Astrophysics and Space Science manuscript No.

(will be inserted by the editor)

Valentí Bosch-Ramon

\title{
Theoretical overview on high-energy emission in microquasars
}

Received: date / Accepted: date

\begin{abstract}
Microquasar (MQ) jets are sites of particle acceleration and synchrotron emission. Such synchrotron radiation has been detected coming from jet regions of different spatial scales, which for the instruments at work nowadays appear as compact radio cores, slightly resolved radio jets, or (very) extended structures (e.g. [1, [2, 3]). Because of the presence of relativistic particles and dense photon, magnetic and matter fields, these outflows are also the best candidates to generate the very high-energy (VHE) gamma-rays detected coming from two of these objects, LS 5039 and LS I +61 303 (4,5, and [6, respectively), and may be contributing significantly to the X-rays emitted from the MQ core (e.g. 7, 8]). In addition, beside electromagnetic radiation, jets at different scales are producing some amount of leptonic and hadronic cosmic rays (CR), and evidences of neutrino production in these objects may be eventually found. In this work, we review on the different physical processes that may be at work in or related to MQ jets. The jet regions capable to produce significant , amounts of emission at different wavelengths have been reduced to the jet base, the jet at scales of the order of the size of the system orbital semi-major axis, the jet middle scales (the resolved radio jets), and the jet termination point. The surroundings of the jet could be sites of multiwavelegnth emission as well, deserving also an insight. We focus on those scenarios, either hadronic or leptonic, in which it seems more plausible to generate both photons from radio to VHE and high-energy neutrinos. We briefly comment as well on the relevance of $\mathrm{MQ}$ as possible contributors to the galactic $\mathrm{CR}$ in the $\mathrm{GeV}-\mathrm{PeV}$ range.
\end{abstract}

Keywords microquasars - jets - Gamma-rays: theory · multiwavelength: theory $\cdot$ neutrinos

V. Bosch-Ramon

Max Planck Institut für Kernphysik, Heidelberg 69117, Germany

E-mail: vbosch@mpi-hd.mpg.de

\section{Introduction}

MQ are binary systems formed by a normal star and a compact object, either a black hole or a neutron star. In these objects, stellar matter is expelled and some fraction of it eventually forms an accretion disk around the compact object from which two relativistic jets are launched in opposite directions. These relativistic jets are the characterizing feature of MQ, namely a subclass of X-ray binaries (XRB), and were observed for the first time in a galactic object at radio frequencies when studying SS 433 (9], 10]). In fact, the term microquasar became popular when extended radio jets were discoverd in 1E 1740.7-2942 similar to those observed in some quasars (11]). We will focus on processes of high energy emission in MQ, and for more general reviews we refer to other works that can be found in the literature (e.g. [1, [12, 13] and [14).

For several years after their discovery, MQ jets were considered basically as low energy emitters, producing electromagnetic radiation at most up to X-rays (e.g. SS 433, 15 ), and they were not generally considered as gammaray emitters above $\sim 1 \mathrm{MeV}$. There were some theoretical efforts to explain/predict emission at higher energies from MQ (see, e.g. [17]), although at that time these objects were not conceived to be a class of gamma-ray emitters of their own.

Paredes and co-workers (18]) proposed the MQ LS 5039, discovered by the same authors, as the counterpart of the high-energy gamma-ray source 3EG J1824-1514, which had been detected by the high-energy gamma-ray instrument EGRET (19). It was suggested in 18,20 that inverse Compton (IC) interactions between radio emitting relativistic electrons in the jet and UV photons from the massive stellar companion could be behind the gammarays detected by EGRET, possibility that was further explored by 21, who concluded that it was feasible though synchrotron self-Compton (SSC) could not be discarded either. In fact, the discovery of LS 5039 and its position coincidence with an EGRET source triggered an intense 
activity in the field of MQ gamma-ray modeling (e.g. [22], 23], 24], 25], 26], etc.). The EGRET source association of this MQ was virtually confirmed by the HESS detection of LS 5039 (4]), establishing it as the first TeV MQ. The recent detection by MAGIC ([6]) of another MQ, LS I +61 303 ([27,28]), has shown that these objects likely form a new population of gamma-ray sources. It is interesting to note that the $\mathrm{TeV}$ emitter nature of LS I +61 303 renders very likely its association with the EGRET source 3EG J0241+6103, proposed by [29].

Beside gamma-ray photons, the production in jets of other high energy particles has been studied. Some MQ jets contain a hadronic component, as has been observed in SS 433 (e.g. 30, 15, 31, 32]). Such hadronic component, in case it were a common MQ jet feature, would contribute to some extent to the galactic $\mathrm{CR}$ in the $\mathrm{GeV}$ range $(33)$. In addition, if these hadrons were accelerated, they may affect significantly the CR spectrum up to $\sim 100 \mathrm{TeV}$ (33, 34, 35]). Furthermore, the presence of relativistic protons in MQ jets, when interacting with dense matter and high energy photon fields, could lead to the production of gamma-rays ([23, 34]) and neutrinos (e.g. 36], 37]).

Next, in Sect. 2 the physical scenario is set up taking into account the present knowledge on MQ; in Sect. 3 , different possible radiative processes occurring in MQ are briefly presented and discussed; finally, in Sect. 4, the conclusions regarding the mechanisms explained here are provided and discussed in the context of the present observational knowledge.

\section{The MQ physical scenario}

The main components of the MQ scenario are the compact object, the stellar companion, the matter lost by the companion star, the accretion disk, and the jet itself. Here, the jet is considered as a conical region through which the emitting relativistic particles flow confined inside. This region contains a magnetic field that is assumed to evolve attached to jet matter, both being close to equipartition. The matter lost by the star is embedding the jet and can form a very dense medium for OB stellar companions up to relatively large distances. The star in these massive systems is as well a bright source of UV photons and generates a powerful magnetic field (a characterization of the magnetic field of a massive star can be found in, e.g., 38]). Since massive systems present some features, lacking in low-mass MQ, that introduce further complexity to the overall emitting scenario, we will attach ourselves to the high-mass MQ case, although many of the discussed processes could take place as well in low-mass objects.

\subsection{Studying high energy emission in the MQ scenario}

Using the prescription for the MQ scenario given above, some estimations are made and presented in this work for the spectral energy distribution (SED) of the radiation that could be produced in MQ different regions. In our approach the jet plays a very important role. Each emitting zone is treated as homogeneous, with a size being similar to the corresponding typical spatial scale. Bremsstrahlung, synchrotron and (Thomson and KleinNishina) IC losses are taken into account for electrons, and convective losses are considered for both electrons and protons, the latter being not affected by radiative losses in most of cases. To set up the jet properties involved in the performed computations, we have adopted the next values of the relevant parameters of the MQ system: a jet Lorentz factor of 1.1; accretion disk luminosity of $10^{36} \mathrm{erg} \mathrm{s}^{-1}$; disk photon energy of $10 \mathrm{keV}$; a stellar companion luminosity of $10^{39} \mathrm{erg} \mathrm{s}^{-1}$; stellar photon energies of $10 \mathrm{eV}$; an orbital semi-major axis of $0.2 \mathrm{AU}$; a stellar wind mass-loss rate of $10^{-6} \mathrm{M}_{\odot} \mathrm{yr}^{-1}$; and a stellar wind velocity of $2 \times 10^{8} \mathrm{~cm} \mathrm{~s}^{-1}$. The jet and the wind velocities can be taken as the convection velocities in the jet and the wind, respectively, which altogether with the region size define the convection timescales. For the jet termination region, a convection velocity of $\sim 3 \times 10^{9} \mathrm{~cm} \mathrm{~s}^{-1}$ has been adopted assuming downstream jet material convects away at the speed of the postshock region. All these physical quantities can be in principle known from observations, and the values adopted here are typical ones from the literature. All the parameters values are given in Table 1, at the top.

There is a small set of parameters which are free because they refer to physical quantities that are not well constrained at the moment. It is taken a jet kinetic luminosity of $10^{36} \mathrm{erg} \mathrm{s}^{-1}$; a injection luminosity of the relativistic particles of $10^{35} \mathrm{erg} \mathrm{s}^{-1}$; a magnetic field $(B)$ of $1 / 10$ the equipartition value along jet, the equipartition value for the termination region and $1 \mathrm{G}$ for the jet surrounding region; a mean jet particle energy dominated by the bulk motion component (i.e. cold-matter dominated jet) even when there is relativistic particle component (concerning the jet average temperature, see below); a minimum Lorentz factor of relativistic electrons of 100; a minimum Lorentz factor of relativistic protons of 1 ; and an acceleration efficiency (times $q B c$ ) of 0.1 . The power-law index of the particle injection spectrum is fixed to 2 . For the interstellar medium density, a value of $10^{3} \mathrm{~cm}^{-3}$ is taken. All these free parameter values have been adopted similar to those typically found in the literature and have been summarized in Table 1 . at the bottom.

\footnotetext{
${ }^{1}$ It is common to assume the existence of a hot cloud in the innermost accretion disk region so-called corona that would present a different spectrum than the accretion disk (see, e.g. [39]). For simplicity, we consider the photon fields generated in the disk and the corona as the same one, with a mean photon energy between those of the disk and corona photons.
} 
The highest energy particle is derived from the acceleration efficiencies in balance with the radiative losses and the accelerator size, leading to $\mathrm{GeV}$ energies for electrons in the jet innermost regions and $>\mathrm{TeV}$ elsewhere; protons are not cooling restricted, being able to reach $>\mathrm{TeV}$ everywhere in the jet (see e.g. [40]). The adopted acceleration efficiency allows for instance to reach $\mathrm{TeV}$ photon production energies for the emitting particles. The minimum electron Lorentz factor is derived from the thermal energy of protons, assuming a proton temperature of $10^{11} \mathrm{~K}$. The magnetic field has been fixed to avoid too strong synchrotron radiation, although its choice is somewhat arbitrary. All this allows to estimate roughly the photon and neutrino production all along the jet. The emission generated by the secondary pairs produced via charged pion and then muon decay is not computed due to the low luminosities of the neutral pion decay gamma component, rendering the secondary radiation negligible when compared with the one of the primary leptonic component. Further issues related to the exact spectral shape and its evolution: photon photon absorption and cascading, variability in target densities or particle injection, geometric effects in the different interaction processes (IC and photon photon absorption), and Doppler boosting in relation to the observer direction, are not studied quantitatively here and deserve a particular treatment source by source out of the scope of this work. SSC is not taken into account either, although it will not affect significantly the electron energy distribution but for the case when the emitter is very compact (i.e. the base of the jet). In such a case, the synchrotron component would be weaker though not much, and the SSC would be similar in flux, although broader in spectral shape, than the disk IC component (see next section).

It is worth before going further to briefly mention the main difference between a low-mass and a high-mass system, the one treated here, concerning gamma-ray emission. As will be seen, the stellar IC is an efficient mechanism to produce significant amounts of $\mathrm{TeV}$ photons, implying that low-mass systems lacking of a strong source of optical/UV photons may not be strong emitters.

\subsection{Further comments on the jet properties}

Relativistic particles plus magnetic field are unlikely to be the only components of the jet. Relativistic particles lose their energy as far as they are convected away in the jet under the present magnetic and photon fields, and they have to be accelerated far away from the compact object when extended radio/X-ray emission is observed. Moreover, emitting particles must be confined to keep the (supposed to be) conical jet shape with a small
Table 1 Parameter values adopted in this work

\begin{tabular}{ll}
\hline Parameter & Value \\
\hline Jet Lorentz factor & 1.1 \\
Accretion disk luminosity & $10^{36} \mathrm{erg} \mathrm{s}^{-1}$ \\
Disk photon energy & $10 \mathrm{keV}^{39}$ \\
Stellar companion luminosity & $10^{39} \mathrm{erg} \mathrm{s}^{-1}$ \\
Stellar photon energy & $10 \mathrm{eV}$ \\
Orbital semi-major axis & $0.2 \mathrm{AU}$ \\
Stellar mass-loss rate & $10^{-6} \mathrm{M}_{\odot} \mathrm{yr}^{-1}$ \\
Stellar wind velocity & $2 \times 10^{8} \mathrm{~cm} \mathrm{~s}^{-1}$ \\
Jet-ISM postshock speed & $3 \times 10^{9} \mathrm{~cm} \mathrm{~s}^{-1}$ \\
\hline Jet kinetic luminosity & $10^{36} \mathrm{erg} \mathrm{s}^{-1}$ \\
Particle injection luminosity & $10^{35} \mathrm{erg} \mathrm{s}^{-1}$ \\
Ratio magnetic field/equipartition value & 0.1 \\
Electron minimum Lorentz factor & 100 \\
Proton minimum Lorentz factor & 1 \\
Acceleration efficiency & 0.1 \\
ISM density & $10^{3} \mathrm{~cm}^{-3}$ \\
\hline
\end{tabular}

opening angle (e.g. SS 433, 31]; LS 5039, 20]). It implies the existence of a non-emitting background outflow that dominates dynamically the whole jet, sometimes called dark jet. We are not concerned on this background here since its detailed physics is not needed for our exploration. The background jet action on the radiative part is taken into account phenomenologically, assuming this background confines and accelerates particles, which is enough at the level of approximation of our exploration.

The dark jet might consist on matter extracted from the inner parts of the accretion disk (strongly ionized matter with temperatures $\sim 10^{7} \mathrm{~K}$ ) and/or the corona (a hot matter halo with temperatures of $\sim 10^{11} \mathrm{~K}$ for protons and $\sim 10^{9} \mathrm{~K}$ for electrons around the compact object; e.g. 41). The mean energy per particle of this matter, redirected from the accretion disk/corona to the jet, would be non relativistic (i.e. cold), kinetically confining the jet due to its low Mach number. This cold matter would prevent efficient relativistic particles to escape via a low enough diffusion coefficient. Another possible type of dark jet would be that formed by a light and relativistic pair beam that would be (re-)energized by magnetic energy dissipation due to instabilities occurring in a surrounding magnetically dominated flow. Such flow would be dynamically dominant and would play the role of the jet confining component. Each case would be associated to different launching mechanisms, accretion vs. black hole rotation (e.g. 42], [43, respectively), although a combination of all these possibilities could be the case (for recent proposals for jet launching, see e.g. [44, 45, 46]). Other on-going debates concern the discrete or continuous nature of the outflows ejected from MQ, and which radio/X-ray states different types of jet are related to. For a recent treatment of the problem, we refer to [47. Due to the simplicity of our approach, we consider the jet as a continuous structure in which acceleration and emission processes can take place at 


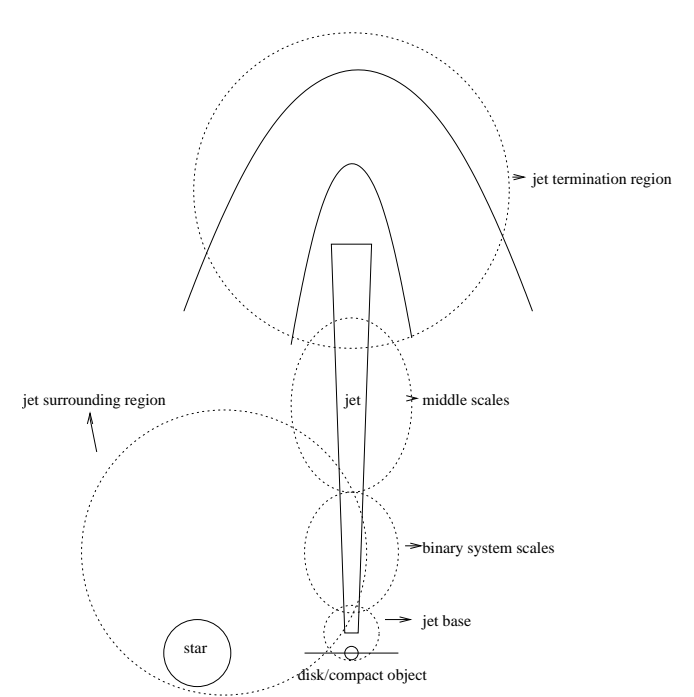

Fig. 1 Sketch of the MQ five considered regions.

different locations. Nevertheless, it is worth noting that the outflow could suffer strongly time-dependent injection, it could be formed by discrete ejections, or it may be a chain of active knots on a background non emitting continuous flow. Such knots could be produced by either internal shocks between different velocity shells, dissipation of energy via magnetic instabilities, or fast Alfven waves moving fast through the background matter (maybe all of them altogether).

\section{The MQ regions}

A MQ can be divided in five regions according to the dominant conditions that affect the radiative processes: the jet base, at 100 Schwarzschild radii from the compact object $\left(\sim 100 R_{\text {Sch }} \sim 10^{8} \mathrm{~cm}\right.$, for a compact object of $\left.\sim 3 \mathrm{M}_{\odot}\right)$; the jet at distances similar to the binary system size, $\sim 3 \times 10^{6} R_{\mathrm{Sch}} 2$; the middle scales, at distances $\sim 10^{9} R_{\mathrm{Sch}}$; the jet termination region, at $\sim 10^{12} R_{\mathrm{Sch}}$; and the regions surrounding the jet, with typical sizes that would depend on the jet environment (i.e. the stellar wind in a massive system). For the jet surrounding region, the size of the emitter is taken that of the binary system. For the remaining regions, the jet size will be taken one fifth of the distance $l$, which may appear somewhat arbitrary but its precise value does not affect significantly our estimates. In Fig. 1, the five regions are shown. In Table 2 we present the sizes and distances to the compact object corresponding to the different mentioned regions.

2 This region would be imposed by the presence of a massive star in the system since in low-mass MQ it is unclear whether the star could be something else than a matter supplier, without effects in the radiation processes.
Table 2 The MQ regions

\begin{tabular}{lll}
\hline Region & distance & size \\
\hline Jet base & $10^{8} \mathrm{~cm}$ & $2 \times 10^{7} \mathrm{~cm}$ \\
Binary system scales & $3 \times 10^{12} \mathrm{~cm}$ & $6 \times 10^{11} \mathrm{~cm}$ \\
Middle scales & $10^{15} \mathrm{~cm}$ & $2 \times 10^{14} \mathrm{~cm}$ \\
Jet termination point & $10^{18} \mathrm{~cm}$ & $2 \times 10^{17} \mathrm{~cm}$ \\
Jet surroundings & $3 \times 10^{12} \mathrm{~cm}$ & $3 \times 10^{12} \mathrm{~cm}$ \\
\hline
\end{tabular}

\subsection{The jet base}

The innermost jet is usually associated to the inner accretion disk, where the jet is formed. This would correspond to a region in AGN jets of $\sim 10^{16} \mathrm{~cm}$, which is observable in some nearby objects, like M 87 or Centaurus $A$ (48] and 49], respectively). Unfortunately, unlike in AGN, the jet base size in MQ is several orders of magnitude away from the present resolution capabilities of the radio interferometers at work. The linear size is proportional to the compact object mass, but the angular size is inversely proportional to the distance, leading to a resolution capability $10^{5}$ times better for the nearest AGN than in case of MQ, preventing to probe this region of the jet for the latter objects.

To compute the emission from leptons and hadrons in the jet base, there are several elements to take into account. These elements are the magnetic field, the accretion disk photon field and the jet hadrons 3 , the last two being much denser there than the stellar photon and matter fields. The mechanisms behind particle acceleration in this region are unclear. If the jet base were magnetically dominated (as considered by e.g. [50]), one of these mechanisms could be magnetic energy dissipation via MHD instabilities, which would accelerate particles (e.g. 51 ). Also, if jet velocities were high enough in the jet base, the dense available photon and matter fields could allow the converter mechanism to take place (52]). Reconnection of large scale magnetic fields in the surrounding corona could inject a non-thermal population of particles in the jet as well (see, e.g., [53] and references therein).

Depending on the dominant conditions, the relevant leptonic radiative mechanisms in the jet base could be synchrotron emission (e.g. [7]), Bremsstrahlung by interacting with jet ions (54), SSC (e.g. [21,55) and IC with corona and/or disk photons (e.g. 56). Regarding hadronic processes, the radiative mechanisms that could produce gamma-rays, neutrinos and, as a by-product, low energy emission from secondary pairs are the interactions of relativistic protons with ions in the jet, and interactions between jet relativistic protons and Xray photons from the disk, the corona or the jet itself (e.g. 57, 36). These proton ion collisions would produce neutral pions $\left(\pi^{0}\right)$ that decay to gamma-rays, and

3 These hadrons may be also disk or corona ions, if mixing with jet relativistic protons takes place. 


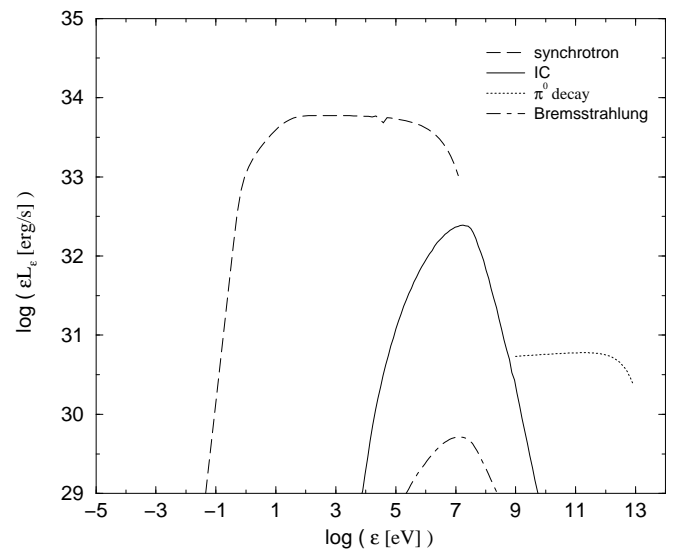

Fig. 2 SED for the synchrotron and IC processes, (protonproton) $\pi^{0}$ decay, and Bremsstrahlung in the jet base under the conditions given in Sect. 2. We note that photon absorption is not considered and only the production SED is presented.

charged pions $\left(\pi^{ \pm}\right)$that decay to muons and neutrinos, the former decaying then to electron-positron pairs and neutrinos. The presence of strong X-ray photon fields renders the possibility of $\leq \mathrm{GeV}$ gamma-ray absorption cascading quite likely (e.g. [16, [58]). In Fig. 2, we show the SED for the synchrotron, IC, (proton-proton) $\pi^{0}$ decay and Bremsstrahlung components computed for the model described in Sect. 2. It is seen that synchrotron is the dominant radiative mechanism, with strongly selfabsorbed radio emission, partially cooled spectrum at high energies, and maximum photon energies reaching hard X-rays. We do not compute neutrino emission, although for the cases when the proton proton gamma-ray component is shown, the corresponding neutrino SED is to be similar (neglecting absorption).

The variability of the radiation coming from the jet base could be very fast due to the involved spatial scales and is beyond the timing capabilities of the present gamma ray instruments.

\subsection{The binary system scales}

At the binary system scales, the jet in radio appears still as core emission, although the corresponding size in an AGN jet would be tens of pc and can be resolved by the present interferometers. In high-mass systems, the magnetic field in the jet, if in equipartition, competes with the stellar photon field concerning lepton cooling. Moreover, convective and adiabatic losses are as well significant for the lepton spectrum at lower energies. The stellar wind at these scales is perhaps a good target for jet proton wind ion collisions, as well as for jet electrons, if wind/jet matter mixing is significant. Plausible mechanisms for these scales to generate relativistic particles are the different versions of the Fermi process: shock diffusive (Fermi I), random scattering (Fermi II) and shear

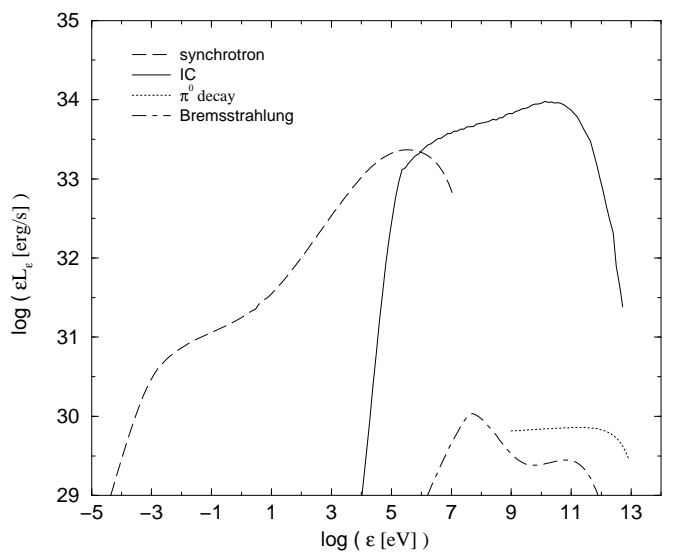

Fig. 3 The same as in Fig. 2 but for the jet emission at binary system scales.

acceleration (e.g. [59, 60 and 61, respectively; see also [40]). Fermi I mechanism could take place due to internal shocks in the jet; Fermi II acceleration could take place if magnetic turbulence is significant enough; shear layer would be a natural outcome of an expanding jet or different jet/medium velocities. Interaction with the stellar wind may also trigger particle acceleration.

From the different elements playing a role at these scales within the microquasar context, possible significant emitting leptonic processes could be synchrotron emission (e.g. 62], 54, [26]), Bremsstrahlung (e.g. [54]), and IC (e.g. 18, 20, 22, 63, 21, 25, 64]). Hadronic processes that may play a significant role at binary system scales could be proton proton collisions with the stellar wind (e.g. 23), which would lead as well to neutrino production (e.g. 65, 36). In Fig. 3, we show the SED for the synchrotron, IC, $\pi^{0}$ decay and Bremsstrahlung components produced in the jet at binary system scales. We note that stellar IC and synchrotron processes com- pete at some extent being both significant. Synchrotron emission is still quite self-absorbed at radio frequencies, and compete with IC at X-rays, which reaches VHE. We recall that photon photon absorption $([66,67)$ and electromagnetic cascading (e.g. [36, 68]) are not computed and only the production spectra are shown.

Variations of high energy emission coming from these jet scales could be detected, and could be linked to orbital changes of the emission and absorption processes (injection of relativistic particles, changes in targets, etc.).

\subsection{The jet middle scales}

MQ jet middle scales are already within the resolution capabilities of the present interferometers though extended radio emission is generally faint. These regions would have a size of $\sim 10 \mathrm{kpc}$ in AGN. In this region of the MQ jet, the outflowing matter is probably overpressing 


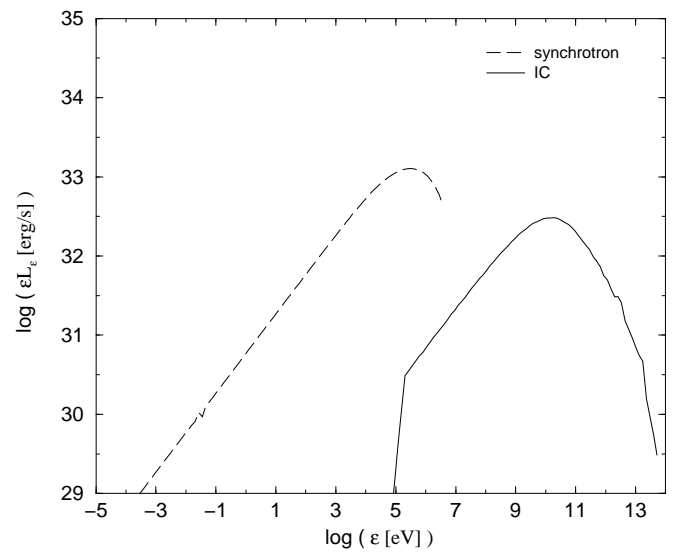

Fig. 4 The SED for the synchrotron and IC components for the jet emission at middle scales.

the surrounding medium and in such a case no significant interaction will be expected, unlike in extragalactic jets. Regarding particle acceleration, although internal shocks may take place still at these distances from the compact object associated perhaps with violent episodes occurring in the jet base, Fermi II type and shear acceleration appear more plausible for a continuous outflow at these scales (something similar could happen in the intra-knot regions of extragalactic jets, see, e.g., 40]).

At the scales concerned here, only synchrotron emission could be significant in persistent jets, and stellar IC seems already a bit inefficient (e.g. [54]) and could be masked by emission coming from inner regions. The particle distribution evolution is likely dominated by convective and adiabatic losses (69). Nevertheless, SSC could still be significant for powerful ejections (e.g. [17). In Fig. [4 we present the computed SED for the synchrotron and IC components of the radiation produced at jet middle scales. Neither Bremsstrahlung nor proton proton collisions are likely to be relevant for the expected low densities of the jet and its environment. Using the parameters provided above, it is seen that synchrotron radio emission is faint and optically thin and dominant losses are convective ones. X-ray emission could be still significant.

The variability of the radiation at this scales could come from long term changes in the system properties, like for instance the mass loss rate of the star.

\subsection{The jet termination region}

At the jet termination point, as in AGN hot spots and radio lobes, two shocks may be formed or become important when the swept ISM inertia starts to affect the

\footnotetext{
4 Such environment is assumed to be similar to the typical galactic ISM, although it could be much more complicated in high-mass systems, since the stellar wind cannot be neglected.
}

jet advance, one moving backwards in the jet (i.e. the reverse shock), and another one moving forward (i.e. forward shock). Fermi I type mechanism seems the most reasonable option under these conditions, although the diffusive and convective efficiency in the downstream regions of the forward/reverse shocks could prevent efficient acceleration to occur. For particles accelerated in such shocks, synchrotron, Bremsstrahlung and IC processes in case of leptons and proton proton collisions in case of hadrons could produce non negligible amounts of radiation. It might be the case as well that hydrodynamical instabilities distorted the jet and mixed jet matter with the ISM without forming a strong shock (for a thorough discussion on this region properties, see [33]). We note that even without shock, some interaction may still take place between jet hadrons and ISM nuclei.

It is difficult to ascertain whether the jet termination region can accelerate particles efficiently. Extended $\mathrm{X}$-ray synchrotron emission has been observed from the MQ XTE J1550-564 likely produced by accelerated electrons in a termination jet shock ([3], see also [70]). Otherwise, extended radio emission at pc scales has been detected, for instance, coming from 1E 1740.7-2942, which could be related to the interaction of the MQ jets with a surrounding molecular cloud ([11]). Other examples of the interaction between a galactic jet and its environment are SS 433 and Cygnus X-1 (71] and [72, respectively). Therefore, although a different morphology has been found for the jet largest scales in AGN and MQ, it could be explained by the differences in scaling of the physical quantities involved in the jet-medium interaction process in galactic and extragalactic jets (e.g. [73]), i.e. one cannot simply scale with for instance the black hole mass, since the environment does not need to be correlated with it (at least in a straightforward manner).

In case particle acceleration and confinement would be efficient, synchrotron radiation could be produced presenting moderate fluxes at few kpc distances (e.g. 74). Hadronic acceleration could take place as well, which could lead to gamma-ray production (e.g. [33]) and secondary leptonic emission if embedded in or close to a dense medium (e.g. 34). It seems that such relativistic hadrons would hardly dominate the CR galactic component in our galaxy (e.g. 35]), although the cold part could still be significant (e.g. [33]). In Fig. [5, we show the SED of the synchrotron, IC, $\pi^{0}$ decay and Bremsstrahlung components for the parameters described above. In the presented situation, synchrotron emission is significant at all wavelengths from radio to hard X-rays, and the other mechanisms are negligible5.

5 It does not imply that it should be the case in general. For high hadronic densities, or for lower convective velocities of the shocked ISM, it could be possible to get higher gammaray fluxes. The parameter setting is probably less constrained for this case than previous ones because observational data is sparse. 


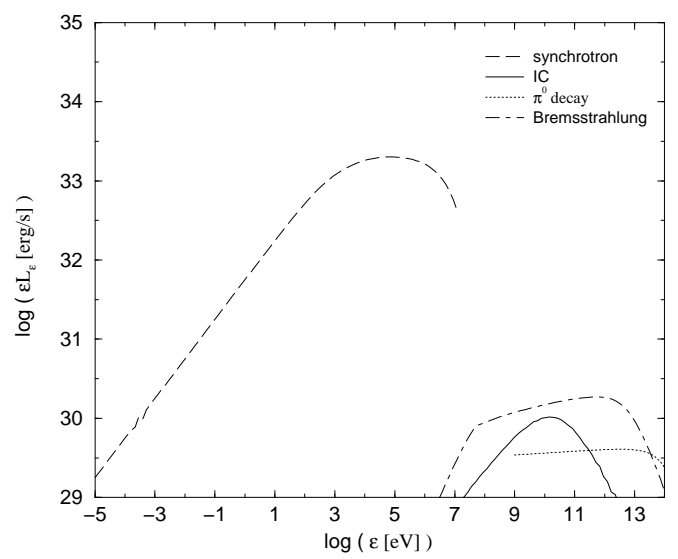

Fig. 5 The same as in Fig. 2 but for the jet termination point radiation.

The timescales at which radiation can change where the jet terminates are large, of at least several years, being difficult to follow.

\subsection{The jet surroundings}

Jets are likely efficient confining relativistic particles. Typically, accelerated particles are considered to reach a maximum energy in jets that, when cooling is inefficient enough, corresponds to a typical diffusion length equal to the size of the jet. Actually, it does not need to be the case all along the jet. Confinement could fail because local changes of the jet conditions, mixing with the surrounding medium, etc. Moreover, for systems in which photon photon opacities are high for a significant fraction of the whole solid angle, as seen from the gamma-ray emitter, great amounts of pairs will be created when gamma-rays interact with the ambient photons outside the jet. All this may lead to the injection of a relativistic population of particles in the jet surroundings, likely with high minimum energies. Perhaps the most interesting case to study is that when particles are injected at binary system scales in a massive system, since particles could be confined within (as secondary particles generated by cascading; see, e.g., 75]). Confined particles would move with the wind allowing for more efficient energy dissipation of this particles, since the convection velocity of the wind is almost two orders of magnitude lower than that of the jet. For leptons, there are significant matter, magnetic and photon fields to interact with, which may lead to significant synchrotron and IC radiation. For hadrons, the wind ions represent a dense target for hadronic interactions which could lead to neutrino and gamma-ray production (e.g. [76, 36]). In Fig. 6, we show the SED synchrotron, IC, $\pi^{0}$ decay and Bremsstrahlung components in the jet surroundings using the parameters given in Sect. 2. In the present scenario, synchrotron emission is significant at

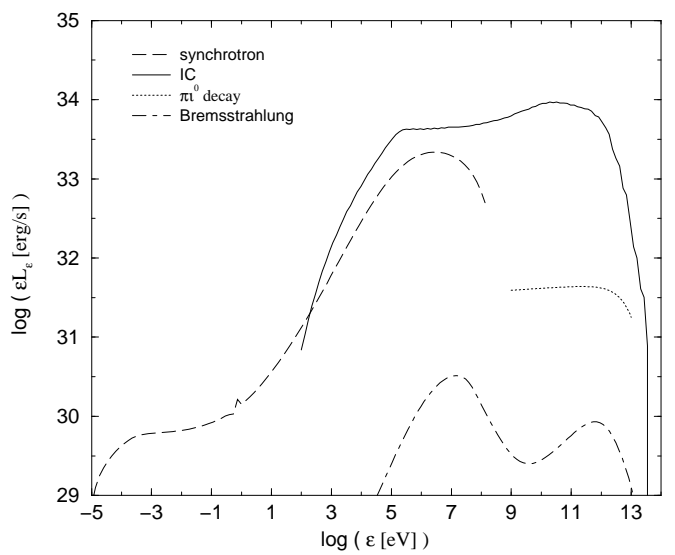

Fig. 6 The same as in Fig. 2 but for the jet surroundings.

$\mathrm{X}$-rays, where competes with IC emission, and it is non negligible either in the radio band though suffer strong self-absorption (and may suffer absorption in the stellar wind) at centimetric wavelengths. IC radiation is also a very efficient process producing gamma-rays, that should be affected as well by photon photon absorption and cascading, which is not considered here.

Regarding variability, it will depend on the energy of particles and the properties of the medium where these particles will be injected. In a high-mass system, orbital variability of the radiation coming from the most energetic particles could take place due to injection changes along the orbit. Low energy particles would present much less variability.

\section{Discussion}

Taking into account all the possible processes described above, let us look at the observational results to compare them with the expectations from the different theoretical models. It is worth noting that it has not been possible till recently, less than one decade ago, to probe high energy radiative processes in MQ because of the lack of data in that energy domain. There were some claims of detection of Cygnus X-3 at gamma-rays by instruments working at VHE and UHE instruments (e.g. [77), although these detections are considered doubtful nowadays by the VHE community. In any case, a significant production of gamma-rays above $1 \mathrm{MeV}$ in $\mathrm{MQ}$ was not thought as plausible. At that time, the main practical reasons behind the difficulties detecting microquasars were that, on one hand, sensitivity was not high enough to reach reliable detections and, on the other, poor angular resolution rendered identification of the counterpart at lower energies a very tough task. However, since the proposal of LS 5039 as an EGRET source (18), MQ scenario started to be explored more actively from the theoretical and the observational points of view by groups coming from different fields (AGN, GRB, pulsars, 
radio stars, XRB, etc.). Nowadays, there are evidences brought by the new instrumentation supporting MQ as a population of gamma-ray sources. The facts behind those evidences can be linked with the described mechanisms that could be at work in MQ.

\section{Large scale jets}

It is well known, as has been noted above, that MQ jets contain relativistic particles emitting synchrotron radio emission at spatial scales that comprehend from those not resolved by the present interferometers to the termination jet region. Moreover, some MQ have shown non-thermal X-ray emission from large-scale jets, which is evidence of the presence of $\mathrm{TeV}$ particles and external shocks (e.g. 3]). Thus, all this tells us that leptonic particles can reach $\mathrm{TeV}$ energies in the jet termination regions. Such $\mathrm{TeV}$ leptons can produce $\mathrm{IC} \mathrm{TeV}$ radiation if a significant source of photons is nearby. In addition, ions are known to be heated in jets up to quite large distances (e.g. [32]), and could be the energy carriers at least in some MQ jets (e.g. [72]). This suggests the possibility of hadronic acceleration and $\pi^{0}$-decay gamma-ray and neutrino production by proton proton collisions, with lower energy photons generated via synchrotron emission from the $\pi^{ \pm}$-decay pairs as by-products. The current upper-limits imposed by HESS and MAGIC on the extended $\mathrm{TeV}$ emission from MQ make the detection of extended neutrino emission quite unlikely for the near future (at such spatial scales, photon photon absorption cannot mask the overall flux of VHE photons, being similar thus to the neutrino one), but extended $\mathrm{TeV}$ emission may still be detected in the near future either by HESS and MAGIC or by HESS- and MAGIC-II.

\section{Variable TeV emission}

Two MQ have been clearly detected at TeV energies: LS 5039 and LS I +61 303 (4] and [6], respectively); both objects also proposed to be EGRET sources (18 and 29, respectively), which has been virtually confirmed by their VHE detections. Concerning the physical processes occurring in microquasars, much information can be obtained from this recent VHE data of MQ. TeV emission from LS I +61 303 has shown to be variable at scales similar to those of the orbital period, pointing to a strong link between the emission processes and the change of the emitter properties along the orbit ([6]). For LS 5039, strong TeV variability, orbital periodicity and absorption effects on the VHE radiation shows that this emission is likely coming from a small region $\leq$ few AU ([5]). Therefore, hadronic and/or leptonic acceleration is probably taking place at binary system scales. In the context of leptonic models, the strong losses and high photon photon internal opacities in the base of the jet (e.g. [54]) will likely prevent that region from being a significant $\mathrm{TeV}$ emitter, pointing to stellar IC and the jet at binary system scales as the radiative process and the emitting region, respectively, behind the VHE radiation. For hadronic processes, radiative losses are not relevant enough in general to limit proton energy, although re- quiring as well very large target densities and kinetic luminosities in relativistic hadrons. Cascading seems unavoidable for a jet base hadronic model. The higher efficiency of IC interaction leaves more room for significant $\mathrm{TeV}$ photon production outside the binary system, although still close to it $(\sim 1 \mathrm{AU})$.

The details concerning the injection particle distribution, or at which extent photon photon opacities affect the final TeV spectrum, are still unknown. Different particle energy distributions, with different evolution and overall shape because different injection location and jet conditions, may be contributing to the final gamma-ray spectrum. Because of the emitting size is not properly settled, it seems not possible at the moment to infer the local production spectrum applying only an absorption or a cascading model. Due to the complexity of leptonic and hadronic mechanisms, it is hardly feasible presently to put forward a method for favoring with strong evidence any of both scenarios but via neutrino detection. Nevertheless, the present detected luminosities in the $\mathrm{TeV}$ regime show that, unless these systems produce all the emission under high photon photon opacities rendering a high neutrino to photon ratio, it will be difficult to detect them even with $\mathrm{km}^{3}$ neutrino detectors (e.g. 36, [37, 78, 79]).

One interesting fact concerning both the X-rays and $\mathrm{TeV}$ gamma-rays is that they appear to be correlated in LS 5039. There is a clear peak and a spectral hardening at the same orbital phase, $\sim 0.8$, in both X-rays and $\mathrm{TeV}$ energies $(8,8,80,[5)$. This could happen as well in LS I +61 303, where the X-ray and the TeV peaks occur at similar phases $\sim 0.5$ ([81, 6]), and there is also a hardening in the X-ray spectrum when X-ray flux increases ([82]). There might be also a strong connection between radio and $\mathrm{TeV}$ photons in these two $\mathrm{TeV}$ MQ. In both systems, most of the radio emission come from scales of several AU, but it is optically thin (in LS I +61 303 when it is not in outburst; 83), which points to moderate magnetic fields and particle densities. Moreover, LS 5039 shows quite steady radio fluxes (84). This former fact, altogether with the compactness of most of the radio emission and the mentioned moderate magnetic field, would imply that the radio emission is to be confined (e.g. a low convective velocity, like that of the stellar wind and unlike the jet one). We note that $\mathrm{TeV}$ emission, likely being of stellar IC origin, requires magnetic fields not far from those of radio emission $(\leq 1 \mathrm{G}$, provided the stellar photon field density is $\sim 500 \mathrm{erg} \mathrm{cm}^{-3}$ ), which may mean that radio and $\mathrm{TeV}$ radiation come from similar regions. However, since $\mathrm{TeV}$ luminosities are slightly smaller than the X-ray luminosity upper-limit and $\mathrm{TeV}$ spectrum is not affected by dominant synchrotron losses, the hypothesis that the same population of electrons produces both X-ray and $\mathrm{TeV}$ photons seems to be ruled out. An interesting additional fact is the constant $200 \mathrm{GeV}$ flux in LS 5039 ([5]), which may be related to the radio flux steadiness. 


\section{Multiparticle future}

Previous discussion shows that a deep understanding of the physics behind the high energy radiation observed in $\mathrm{MQ}$ requires of the combination of different processes taking into account particle acceleration, radiation mechanisms, photon photon absorption and cascading, and plasma physics. Nevertheless, this approximation to the problem cannot be done at once, and a step by step study is to be applied accounting for all the existent (and forthcoming) data in combination with theoretical insights on the relevant processes and emitting regions. It is clear that multiwavelength and multiparticle approaches, plus deeper modeling, are indeed the future of the MQ high energy field.

Acknowledgements We are grateful to Josep M. Paredes and Marc Ribó for a thorough reading of the manuscript. We thank also Felix Aharonian, Dmitry Khangulyan and Evgeny Derishev for fruitful discussion that has benefited this work. V.B-R. acknowledges partial support by DGI of the Ministerio de Educación y Ciencia (Spain) under grant AYA2004-07171-C02-01. The Max-Planck-Institut für Kernphysik is thanked for its support and kind hospitality.

\section{References}

1. Mirabel I.F., Rodríguez L.F.: Sources of Relativistic Jets in the Galaxy. ARA\&A 37, 409-443 (1999)

2. Fender R.P.: Powerful jets from black hole X-ray binaries in low/hard X-ray states. MNRAS 322, 31-42 (2001)

3. Corbel S., Fender R.P., Tzioumis A.K., et al.: Large-Scale, Decelerating, Relativistic X-ray Jets from the Microquasar XTE J1550-564. Science 298, 196-199 (2002)

4. Aharonian F. A. et al.: Discovery of Very High Energy Gamma Rays Associated with an X-ray Binary. Science 309, 746-749 (2005)

5. Aharonian F. A. et al.: 3.9 day orbital modulation in the $\mathrm{TeV}$ gamma-ray flux and spectrum from the X-ray binary LS 5039. A\&A, accepted, astro-ph/0607192 (2006a)

6. Albert et al.: Variable Very-High-Energy Gamma-Ray Emission from the Microquasar LS I +61 303. Science 312, 1771-1773 (2006)

7. Markoff S., Falcke H., Fender R.: A jet model for the broadband spectrum of XTE J1118+480. Synchrotron emission from radio to X-rays in the Low/Hard spectral state. A\&A 372, 25-28 (2001)

8. Bosch-Ramon, V., Paredes, J. M., Ribó, M. et al.: Orbital X-Ray Variability of the Microquasar LS 5039. ApJ 628, 388-394 (2005a)

9. Spencer, R. E.: A radio jet in SS433. Nature 282, 483-484 (1979)

10. Hjellming, R. M., Johnston, K. J.: An analysis of the proper motions of SS 433 radio jets. ApJ 246, 141-146 (1981)

11. Mirabel I.F., Rodriguez L.F., Cordier B., Paul J., Lebrun F.: A double-sided radio jet from the compact Galactic Centre annihilator 1E 140.7-2942. Nature 358, 215-217 (1992)

12. Ribó M.: Discovery and study of the microquasar LS 5039 and a search for new microquasars. Ph.D. Thesis, Universitat de Barcelona (2002)

13. Paredes, J. M.: Microquasars as High-energy Gamma-ray Sources. ChJAA 5 120-132 (2005)

14. Mirabel, F.: Microquasars. Proceedings of these conference (2006)
15. Kotani, T., Kawai, N., Matsuoka, M., Brinkmann, W.: Iron-Line Diagnostics of the Jets of SS 433. PASJ 48, 619629 (1996) Nature 266, 512-513 (1977)

16. Akharonian, F. A., Vardanian, V. V.: On the origin of soft gamma-rays from Cyg X-1. Ap\&SS 115, 31-37 (1985) A\&A, 236, 175-179 (1990)

17. Atoyan, A. M., Aharonian, F. A.: Modelling of the nonthermal flares in the Galactic microquasar GRS 1915+105. MNRAS 302, 253-276 (1999)

18. Paredes J.M., Martí J., Ribó M., Massi M.: Discovery of a High-Energy Gamma-Ray-Emitting Persistent Microquasar. Science 288, 2340-2342 (2000)

19. Hartman, R. C., Bertsch, D. L., Bloom, S. D: The Third EGRET Catalog of High-Energy Gamma-Ray Sources. ApJSS 123, 79-202 (1999)

20. Paredes, J. M., Ribó, M., Martí, J.: Confirmation of persistent radio jets in the microquasar LS 5039. A\&A $\mathbf{3 9 3}$, L99-102 (2002)

21. Bosch-Ramon, V., Paredes, J. M.: A numerical model for the gamma-ray emission of the microquasar LS 5039. A\&A 417, 1075-1081 (2004a)

22. Kaufman Bernadó M.M., Romero G.E., Mirabel I.F.: Precessing microblazars and unidentified gamma-ray sources. A\&A, 385, L10-L13 (2002)

23. Romero, G. E., Torres, D. F., Kaufman Bernadó, M. M., Mirabel, I. F.: Hadronic gamma-ray emission from windy microquasars. A\&A 410, L1-L4 (2003)

24. Bosch-Ramon, V., Romero, G. E., Paredes, J. M.: Highmass microquasars and low-latitude gamma-ray sources. A\&A 429, 267-276 (2005b)

25. Dermer, C., Böttcher, M.: Gamma Rays from Compton Scattering in the Jets of Microquasars: Application to LS 5039. ApJ 643, 1081-1097 (2006)

26. Paredes, J. M., Bosch-Ramon, V., Romero, G. E.: Spectral energy distribution of the gamma-ray microquasar LS 5039. A\&A 451, 259-266 (2006)

27. Massi, M., Ribó, M., Paredes, J. M., Peracaula, M., Estalella, R.: One-sided jet at milliarcsecond scales in LS I +61 303. A\&A 376, 217-223 (2001)

28. Massi, M.; Ribó, M.; Paredes, J. M. et al.: Hints for a fast precessing relativistic radio jet in LS I +61 303. A\&A 414, L1-L4 (2004)

29. Kniffen D.A., Alberts W.C.K., Bertsch D.L., et al.: EGRET Observations of the Gamma-Ray Source 2CG 135+01. ApJ 486, 126 (1997)

30. Margon, B., Ann N. Y. Acad. Sci. 336, 550 (1980)

31. Marshall, H. L., Canizares, C. R., Schulz, N. S.: The High-Resolution X-Ray Spectrum of SS 433 Using the Chandra HETGS. ApJ 564, 941-952 (2002)

32. Migliari, S., Fender, R., Mndez, M.: Iron Emission Lines from Extended X-ray Jets in SS 433: Reheating of Atomic Nuclei. Science 297, 1673-1676 (2002)

33. Heinz, S., Sunyaev, R.: Cosmic rays from microquasars: A narrow component to the CR spectrum?. A\&A 390, 751-766 (2002)

34. Bosch-Ramon, V., Aharonian, F. A., Paredes, J. M.: Electromagnetic radiation initiated by hadronic jets from microquasars in the ISM. A\&A 432, 609-618 (2005c)

35. Fender, R. P., Maccarone, T. J., van Kesteren, Z.: Energization of interstellar media and cosmic ray production by jets from X-ray binaries. MNRAS 360, 1085-1090 (2005)

36. Aharonian, F. A., Anchordoqui, L. A., Khangulyan, D., Montaruli, T. Microquasar LS 5039: a TeV gamma-ray emitter and a potential TeV neutrino source. Proceedings of TAUP 2005: The 9th International Conference on Topics in Astroparticle Physics and Underground Physics, 39, 408-415, Journal of physics: conference series, Zaragoza, Spain (2006b)

37. Christiansen, H. R., Orellana, M., Romero, G. E.: Highenergy neutrino emission from x-ray binaries. PhRvD 73, 063012 (2006) 
38. Usov, V. V., Melrose, D. B.: X-ray emission from single magnetic early-type stars. ApJ 395, 575-581 (1992)

39. Esin, A. A., McClintock, J. E., Narayan, R.: Advectiondominated Accretion and the Spectral States of Black Hole X-Ray Binaries: Application to Nova MUSCAE 1991. ApJ 489, 865 (1997)

40. Rieger, F. M., Bosch-Ramon, V., Duffy, D.: Particle acceleration in astrophysical jet sources. Proceedings of these conference (2006)

41. Narayan, R., Yi, I.: Advection-dominated Accretion: Underfed Black Holes and Neutron Stars. ApJ 452, 710 (1995)

42. Blandford, R. D., Payne, D. G.: Hydromagnetic flows from accretion discs and the production of radio jets. MNRAS 199, 883-903 (1982)

43. Blandford, R. D., Znajek, R. L.: Electromagnetic extraction of energy from Kerr black holes. MNRAS 179, 433456 (1977)

44. Meier, D.: The theory and simulation of relativistic jet formation: towards a unified model for micro- and macroquasars. NewA Rev., 47, 667-672 (2003)

45. Hujeirat, A.: A model for electromagnetic extraction of rotational energy and formation of accretion-powered jets in radio galaxies. A\&A 416, 423-435 (2004)

46. Ferreira, J., Petrucci, P. O., Henri, G., Saug, L., Pelletier, G.: A unified accretion-ejection paradigm for black hole X-ray binaries. I. The dynamical constituents. A\&A 447, 813-825 (2006)

47. Fender, R. P., Belloni, T. M., Gallo, E.: Towards a unified model for black hole X-ray binary jets. MNRAS 355, 1105-1118 (2004)

48. Junor, W., Biretta, J. A., Livio, M.: Formation of the radio jet in M87 at 100 Schwarzschild radii from the central black hole. Nature, 401, 891-892 (1999)

49. Horiuchi, S., Meier, D. L.; Preston, R. A.; Tingay, S. J.: Ten Milliparsec-Scale Structure of the Nucleus Region in Centaurus A. PASJ 58, 211-216 (2006)

50. Sikora, M., Begelman, M. C., M., Greg M., Lasota, J. P.: Are Quasar Jets Dominated by Poynting Flux?. ApJ 625, $72-77(2005)$

51. Zenitani, S., Hoshino, M.: The Generation of Nonthermal Particles in the Relativistic Magnetic Reconnection of Pair Plasmas. ApJ 562, 63-66 (2001)

52. Derishev, E. V., Aharonian, F. A., Kocharovsky, V. V., Kocharovsky, Vl. V.: Particle acceleration through multiple conversions from a charged into a neutral state and back. PvRvD 68, 043003 (2003)

53. Gierliński, M., Done, C.: The X-ray/gamma-ray spectrum of XTE J1550-564 in the very high state. MNRAS 342, 1083-1092 (2003)

54. Bosch-Ramon, V., Romero, G E., Paredes, J. M.: A broadband leptonic model for gamma-ray emitting microquasars. A\&A 447, 263-276 (2006)

55. Bosch-Ramon, V., Paredes, J. M.: gamma-ray emission from microquasars: A numerical model for LS I +61 303. A\&A 425,1069-1074 (2004b)

56. Romero, G. E., Kaufman Bernadó, M. M., Mirabel, I. F.: Recurrent microblazar activity in Cygnus X-1?. A\&A 393 , L61-L64 (2002)

57. Levinson, A., Waxman, E.: Probing Microquasars with TeV Neutrinos. PhRvL 87, 171101 (2001)

58. Wu, M., Zhang, C. S.; Li, T. P.: Absorption of gammarays by X-ray photons around an accretion binary source. Ap\&SS 204, 141-153 (1993)

59. Drury, L. O.: An introduction to the theory of diffusive shock acceleration of energetic particles in tenuous plasmas. RPPh 46, 973-1027 (1983)

60. Fermi, E.: On the Origin of the Cosmic Radiation. PhysRev 75, 1169-1174 (1949)

61. Rieger, F. M.; Duffy, P.: Shear Acceleration in Relativistic Astrophysical Jets. ApJ 617, 155-161 (2004)
62. Yuan, F.; Cui, W.; Narayan, R.: An Accretion-Jet Model for Black Hole Binaries: Interpreting the Spectral and Timing Features of XTE J1118+480. ApJ 620, 905-914 (2005)

63. Georganopoulos, M., Aharonian, F. A., Kirk, J. G.: External Compton emission from relativistic jets in Galactic black hole candidates and ultraluminous X-ray sources. A\&A 388, L25-L28 (2002)

64. Gupta, S., Böttcher, M.: A Time Dependent Leptonic Model for Microquasar Jets: Application to LSI 61303 ApJ, in press (2006)

65. Romero, G. E., Orellana, M.: Gamma-ray and neutrino emission from misaligned microquasars. A\&A 439, 237$244(2005)$

66. Böttcher, M., Dermer, C.: Photon-Photon Absorption of Very High Energy Gamma Rays from Microquasars: Application to LS 5039 ApJ, 634, L81-L84 (2005)

67. Dubus, G.: Gamma-ray absorption in massive X-ray binaries. A\&A 451, 9-18 (2006)

68. Romero, G. E., Orellana, M., Christiansen, H. R.: Highenergy gamma-ray and neutrino emission from the microquasar LS I +61 303. Proceedings of these conference (2006)

69. van der Laan, H.: A Model for Variable Extragalactic Radio Sources. Nature 211, 1131 (1966)

70. Wang, X. Y., Dai, Z. G., Lu, T.: External Shock Model for the Large-Scale, Relativistic X-Ray Jets from the Microquasar XTE J1550-564. ApJ 592, 347-353 (2003)

71. Dubner, G. M., Holdaway, M., Goss, W. M., Mirabel, I. F.: A High-Resolution Radio Study of the W50-SS 433 System and the Surrounding Medium. AJ 116, 1842-1855 (1998)

72. Gallo, E., Fender, R., Kaiser, C.: A dark jet dominates the power output of the stellar black hole Cygnus X-1. Nature 436, 819-821 (2005)

73. Heinz, S.: Radio lobe dynamics and the environment of microquasars. A\&A 388, 40-43 (2002)

74. Bosch-Ramon, V.: Broadband emission from high-energy processes in microquasars. Ph.D. Thesis, Universitat de Barcelona (2006)

75. Bednarek, W.: Inverse Compton e-p pair cascade model for the gamma-ray production in massive binary LS I +61 303. MNRAS accepted for publication, astro-ph/0606421 (2006)

76. Bednarek, W.: TeV Neutrinos from Microquasars in Compact Massive Binaries. ApJ 631, 466-470 (2005)

77. Chadwick, P. M., Dipper, N. A., Dowthwaite, J. C., Gibson, A. I., Harrison, A. B.: A 12.6-ms pulsar in Cygnus X-3. Nature 318, 642-644 (1985)

78. Torres, D. F., Halzen, F.: LS I +61 303 as a potential neutrino source on the light of MAGIC results. A\&A, submitted astro-ph/0607368

79. Böttcher, M.: Constraints on the Geometry of the VHE Emission in LS 5039 from Photon-Photon Deabsorption APh, submitted (2006)

80. Casares, J., Ribó, M., Ribas, I.: A possible black hole in the gamma-ray microquasar LS 5039. MNRAS 364, 899908 (2005)

81. Taylor, A. R., Young, G., Peracaula, M., Kenny, H. T., Gregory, P. C.: An X-ray outburst. from the radio emitting X-ray binary LS I +61 303. A\&A 305, 817 (1996)

82. Sidoli, L., Pellizzoni A., Vercellone S.: XMM-Newton observation of a spectral state transition in the peculiar radio/X-ray/gamma-ray source LS I +61 303. A\&A accepted astro-ph/0606722 (2006)

83. Ray, P. S., Foster, R. S., Waltman, E. B., Tavani, M., Ghigo, F. D.: Long-Term Flux Monitoring of LS I +61 303 at 2.25 and $8.3 \mathrm{GHz}$. ApJ 491, 381 (1997)

84. Ribó, M., Reig, P., Martí, J., Paredes, J. M.: X-ray and radio observations of RX J1826.2-1450/LS 5039. A\&A 347, 518-523 (1999) 